\title{
Robotic-Assisted Device in Posterior Spinal Fusion for a High Risk Thoraculombar Fracture in Ankylosing Spondylitis
}

\author{
Ali Suliman, Ronit Wollstein, Benjamin Bernfeld, Alexander Bruskin \\ The Department of Orthopedic Surgery, Division of Spine Surgery, Carmel Lady Davis Medical Center, Haifa, Israel
}

Fractures in ankylosing spondylitis (AS) are often difficult to treat and surgical treatment may be fraught with complications. We describe the use of a robotic-assisted device in the surgical treatment of an unstable L1 fracture in an elderly patient with chronic lymphocytic leukemia and AS. The postoperative course was uneventful and the patient was discharged after 3 days. The use of a robotic-assisted device in spine surgery is particularly indicated in difficult high risk cases.

Keywords: Ankylosing spondylitis; Posterior spine fusion; Robotic assisted

\section{Introduction}

Fractures in ankylosing spondylitis (AS) are often difficult to identify and treat. Vertebral osteoporosis often associated with the condition can weaken the spine as well as contribute to the risk of injury, and the brittle nature of the spine predisposes it to fracture with minor trauma [1]. Because the vast majority of these injuries are unstable and neurological manifestations are more common, surgery is often deemed necessary [2]. Complications increase with age and the complexity of background disease [2]. Robotic-assisted devices are increasingly used for spine fusion in the attempt to minimize radiation exposure while maximizing the accuracy of screw placement. The use of this technology has been shown to decrease opioid use in post-surgery with decreased hospitalization time and lower postoperative complication rate [3]. We describe a case of an L1 fracture in an elderly patient suffering from AS that was successfully treated with pos- terior fusion using a robotic-assisted device. The patient presented with minimal complications in the postoperative period.

\section{Technical Note}

An 81-year-old male suffering from AS presented to our emergency department with acute onset of severe lower back pain without a history of trauma. Past medical history and review of symptoms included hypertension, diabetes and chronic lymphocytic leukemia (CLL). The patient did not describe any preexisting radicular symptoms. On admission, the patient had a body mass index of $27 \mathrm{~kg} / \mathrm{m}^{2}$. Physical examination revealed no underlying deformity, minimal range of motion and tenderness over the spinous processes of the thoracolumbar spine. There were no neurovascular abnormalities. His clinical examination was remarkable for severe pain with every movement, even in bed, and the constant requirement of

Received Jul 4, 2013; Revised Sep 26, 2013; Accepted Sep 29, 2013

Corresponding author: Ronit Wollstein

The Department of Orthopedic Surgery, Division of Spine Surgery, Carmel Lady Davis Medical Center,

7 Michal St., Haifa, Israel

Tel: +972-4-825-0276, Fax: +972-4-825-0272, E-mail: ronitwollstein@gmail.com 
large amounts of pain medication. His initial hemoglobin ( $\mathrm{Hgb})$ level was $10.6 \mathrm{~g} / \mathrm{dL}$ and initial white blood cell count was $21,000 \times 109 / \mathrm{L}$.

Initial radiographs demonstrated AS with a bamboo spine and a suspected fracture of L1. The bone mineral density of the involved vertebra had not been evaluated prior to the injury. A computed tomography (CT) scan confirmed a new unstable compression fracture of L1. This was a chance like fracture through the disc space (Figs. 1, 2).

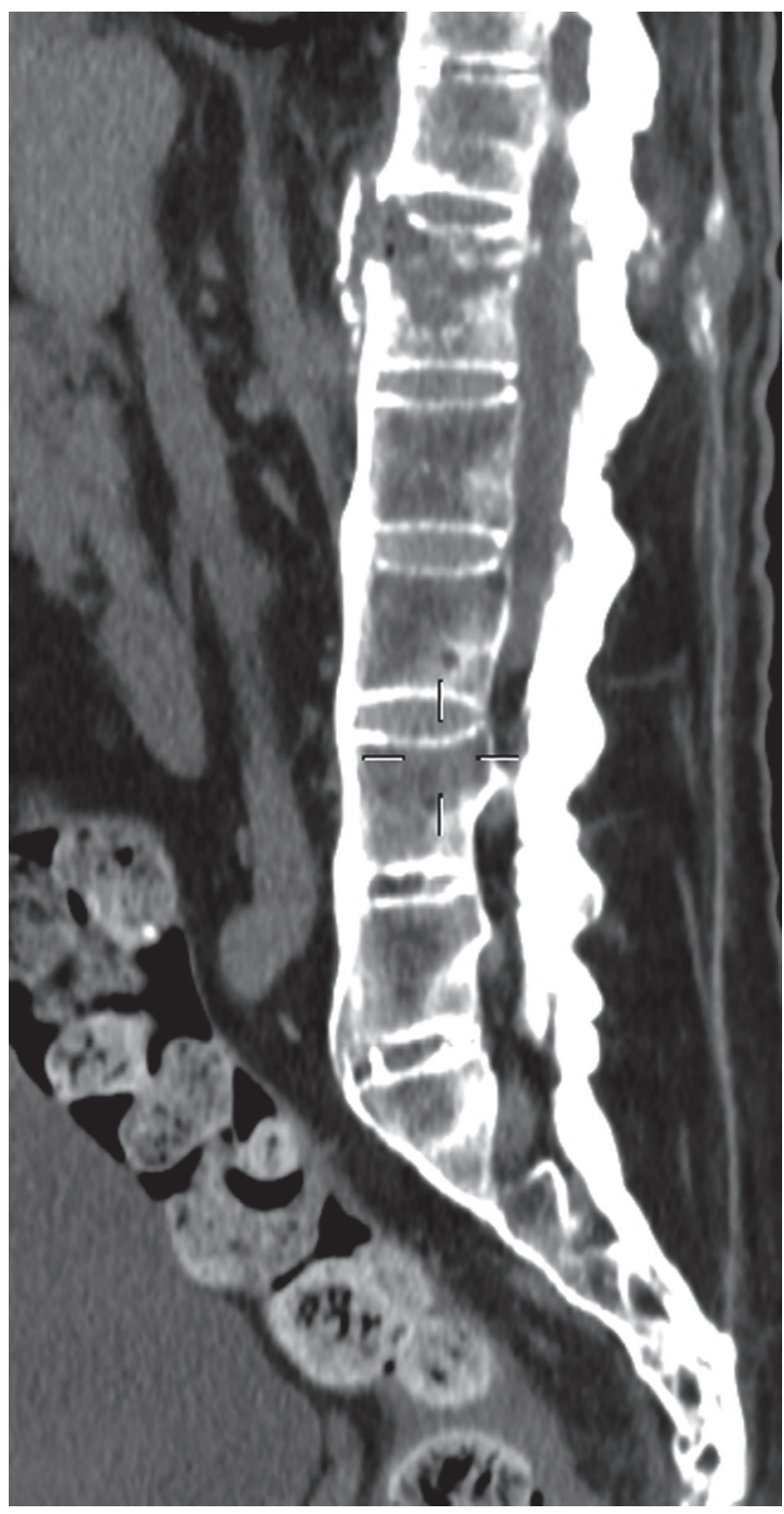

Fig. 1. Computed tomography of the lumbar spine in the sagittal plane demonstrating the fracture.

\section{Technique}

A new CT MAZOR was used for the robot-spine assist device. The surgery requires a preoperative CT scan that is uploaded onto the robot's computer, and the program then calculates the exact 3-dimensional plan. During preparation for surgery, the robot is placed near the patient by directly anchoring it to the spine of the patient. For this process, initial fluoroscopic images are obtained. The robot is approximately 11.4 centimeters high and 6 centimeters wide, with a small arm attached. The robot bends and rotates in order to place its arm on the spine in the specific location and trajectory. This process is timeconsuming and in our experience, using the roboticassisted device lengthened the procedure time to an average of 3 hours and 24 minutes for a one level fusion. For

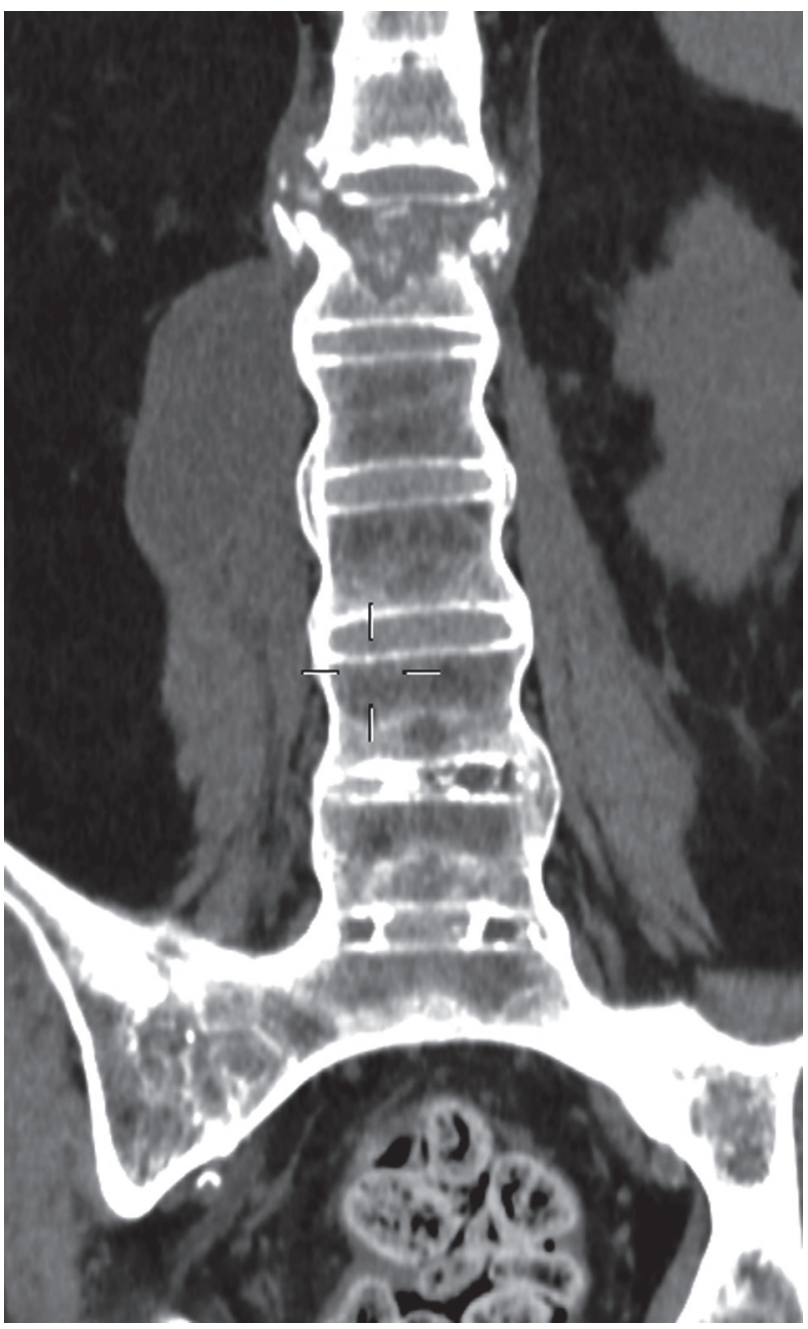

Fig. 2. Computed tomography of the lumbar spine in the coronal plane demonstrating the fracture. 
registration, anatomical landmarks, such as the tips of the transverse process and spinous process, are chosen on the images obtained. A probe is used to identify the pre-selected points on each vertebrae to be instrumented (paired point matching). This registration process correlates the patient's anatomy to the images obtained preoperatively. Accurate registration is confirmed through verifying that a probed point on the exposed spine correlates with a similar location on the image. The size of the incision in this case was about $10 \mathrm{~cm}$ long.

In this case, planning of the operation was performed

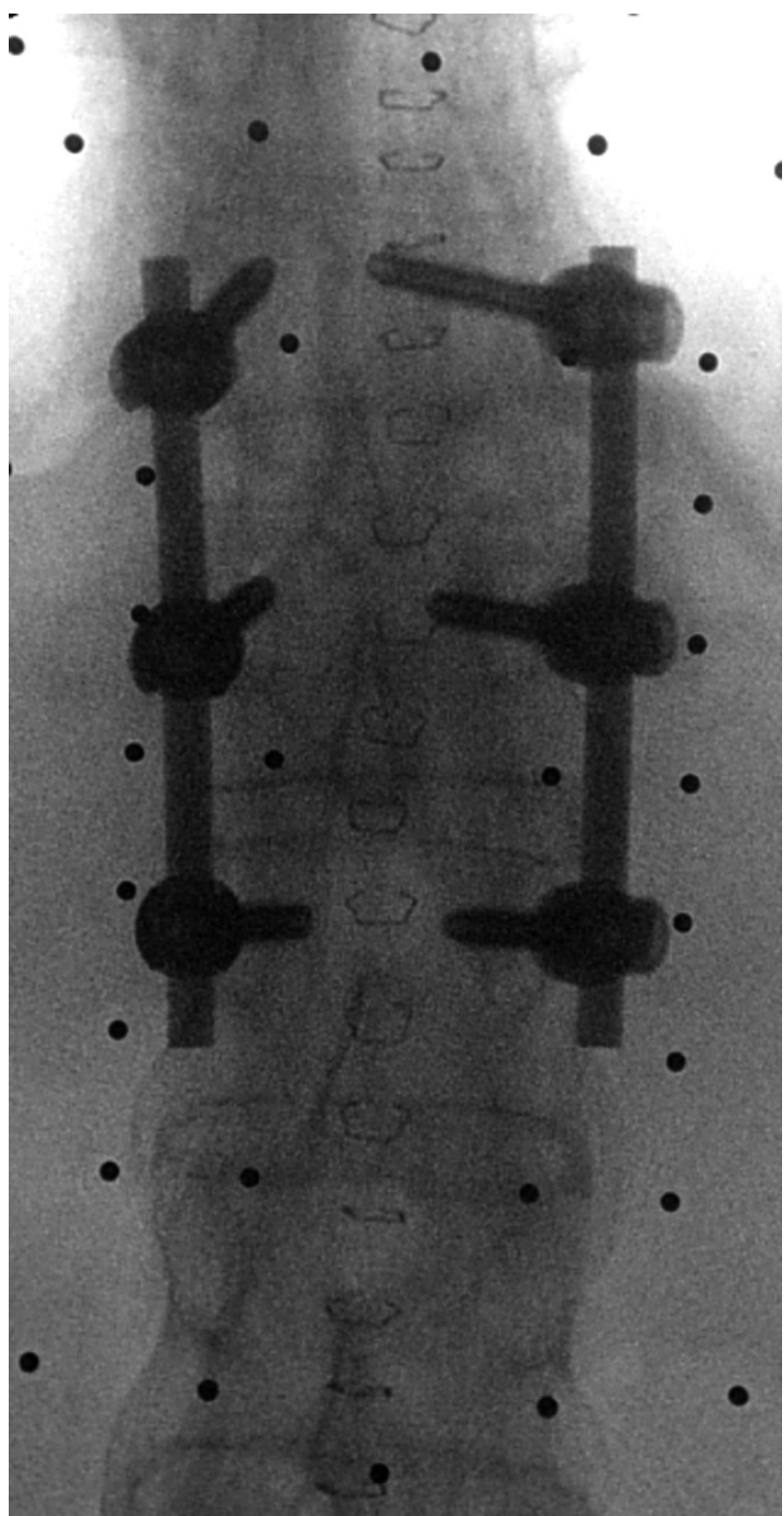

Fig. 3. Intraoperative fluoroscopic image of the spine after fixation posteroanterior. a day before the surgery, consisting of posterior spinal fusion from T12 to L3. The patient was operated on in a prone position. After positioning, a posterior exposure was performed at the levels of T12-L3. Calibration of the device was performed using two fluoroscopic radiographs in order to define the C-arm to the spine assist device; then, registration was performed using another two radiographs (anteroposterior and oblique in 60 degrees) in order to match the patient to the CT Mazor on the spine assist device. The robot was positioned on the spinous process of T12 by a clamp. A posterior spinal fusion T12L2 was performed utilizing six pedicular screws $(6.25 \times 45$ $\mathrm{mm}$ ) and two rods (Figs. 3-5). Despite planning for the fusion of the four levels, the surgeons' impression during

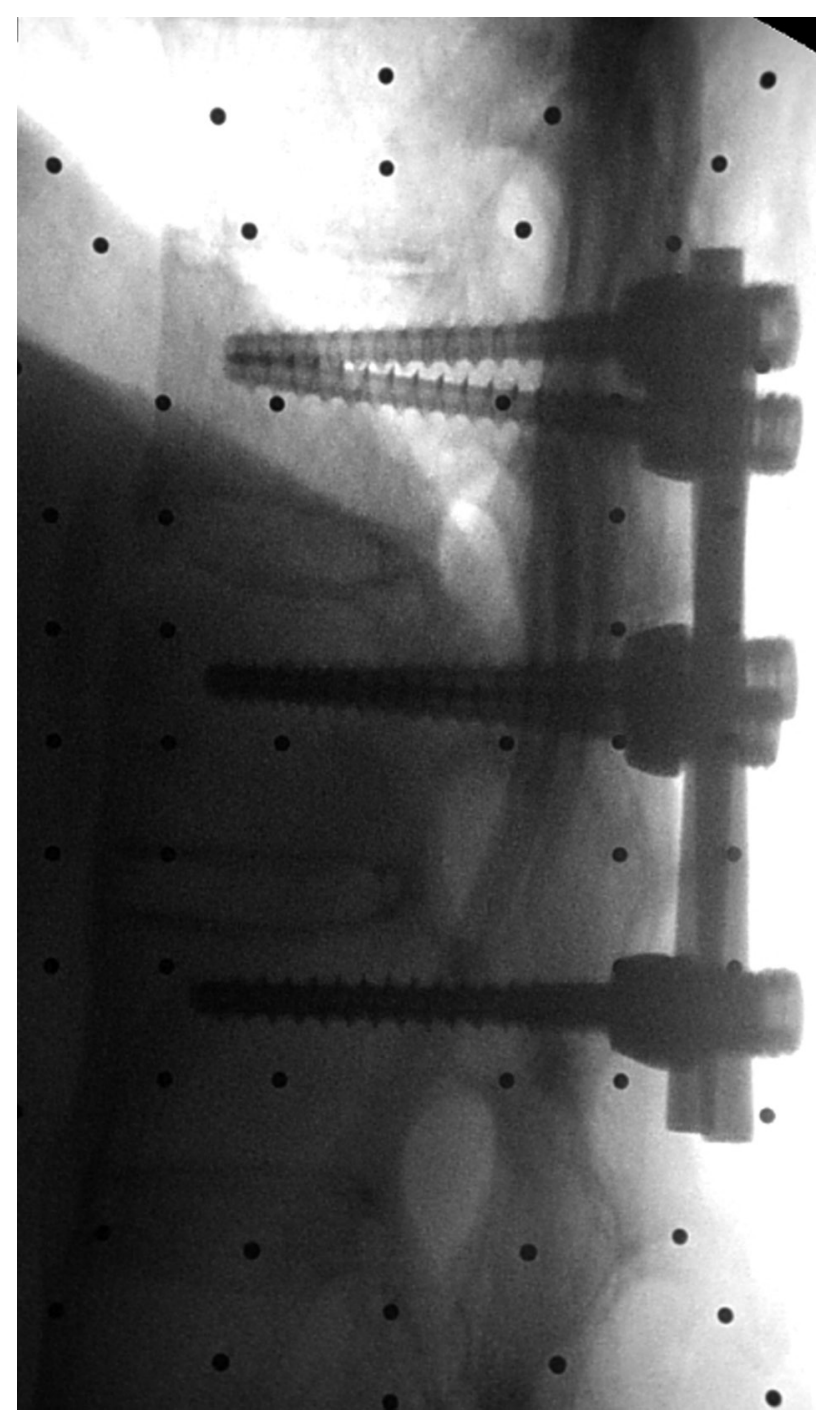

Fig. 4. Intraoperative fluoroscopic image of the spine after fixation lateral. 
the surgery was that the spine segments did not move at all due to AS and possibly degenerative changes in the spine; hence, we decided therefore to fuse only a short segment. The operative time was 2 hours.

\section{Postoperative course}

On the day after the surgery, the patient was allowed to walk using a dorsolumbar spinal brace and with the help of a physical therapist. The patient did have lower postoperative Hgb levels: $9.5 \mathrm{~g} / \mathrm{dL}$, and was therefore given a unit of packed red blood cells with subsequent improvement in $\mathrm{Hgb}$ to $10.5 \mathrm{~g} / \mathrm{dL}$. The patient did not have any other complications following surgery; no fever, problems with coagulation, electrolyte abnormalities or neurovascular compromise; further, white blood cell count remained stable. Patient mobility progressed as expected. He was discharged on day 3 following the surgery. The fracture was healed 14 months after surgery.

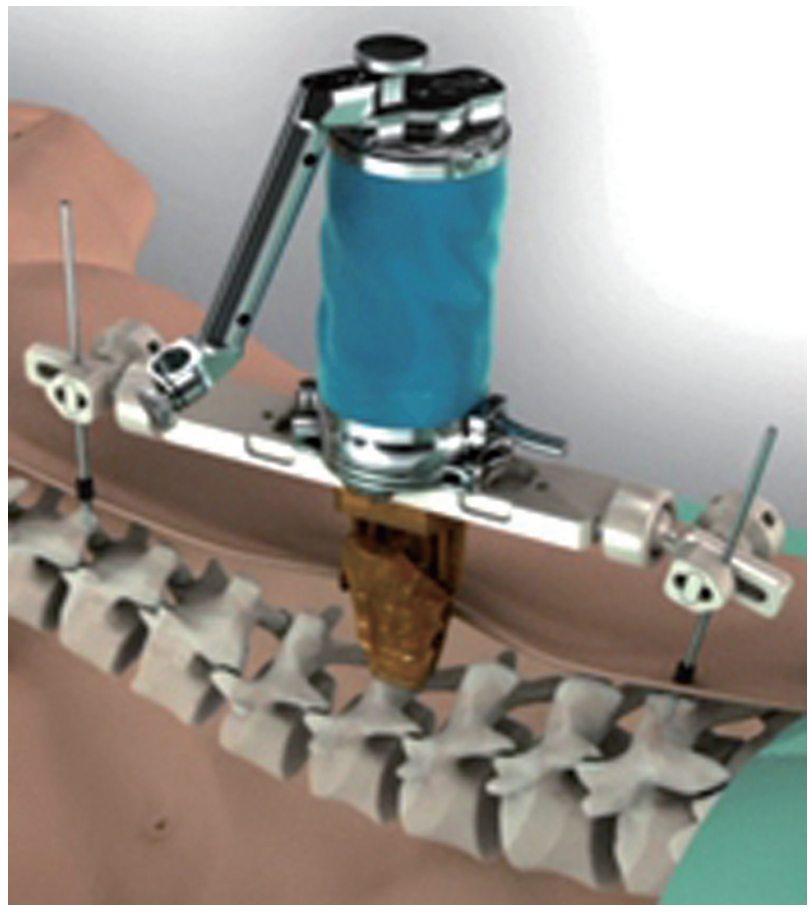

Fig. 5. The robot as it looks when it is clamped onto the spine. The spine is clamped on an adjacent uninvolved vertebra. At this point, registration is performed to link the computed tomography scan to the vertebrae. This is accomplished using two radiographs; one at 60 degrees and one at true anteroposterior seeing an attached threedimensional target completely in both views. Another assessment is then performed for each vertebra to make sure the computer and the vertebrae are aligned, after which the platform is attached. The robot is then attached to the platform in order to allow for accurate insertion of the screws.
The patient was followed up after surgery in the outpatient clinic. Postoperative radiographs were taken at 3 months and at 1 year after the fusion was healed clinically and radiographically (Fig. 6).

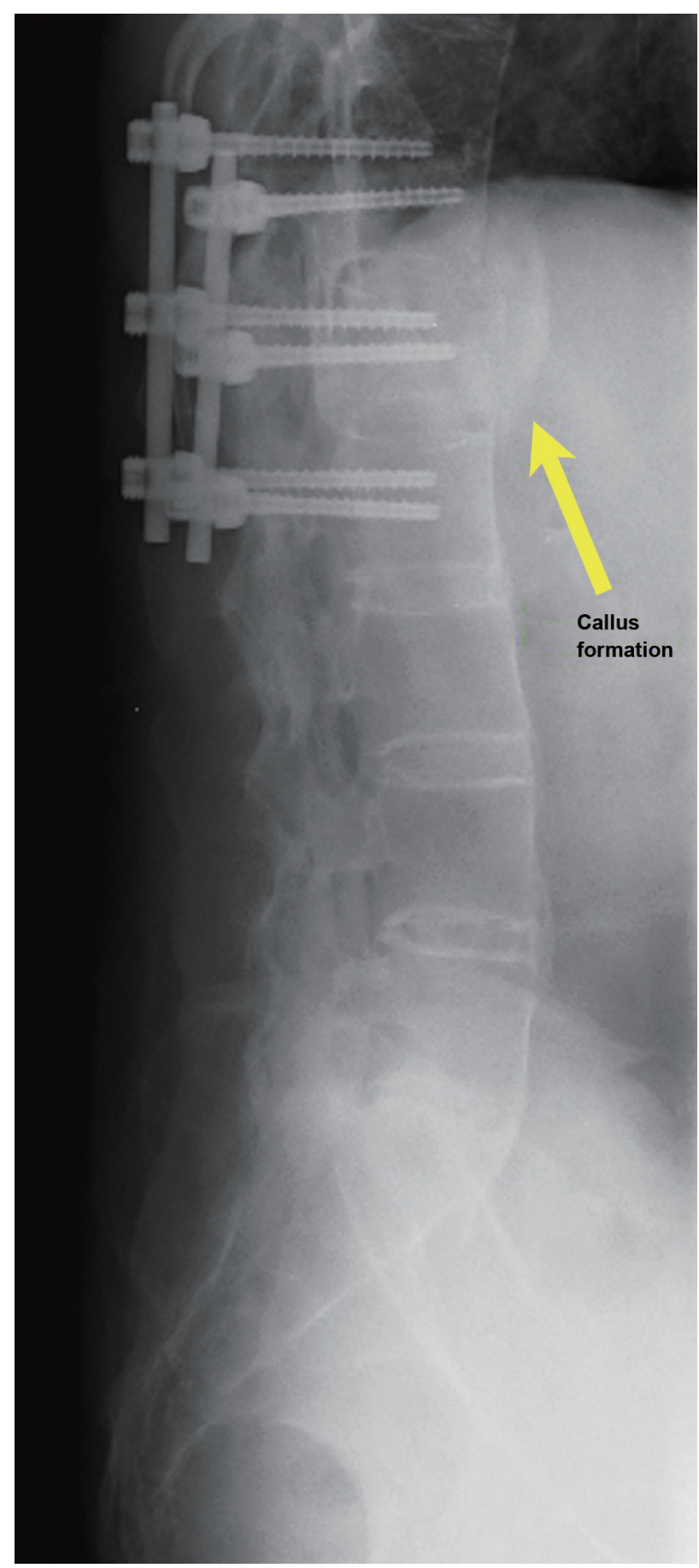

Fig. 6. Lateral view of the spine at one year showing callus formation (arrow). 


\section{Discussion}

Elderly patients with AS and acute fractures have been shown to have better results when treated surgically [4]. Caron et al. [2], in their large retrospective review, showed a mortality rate of $51 \%$ in the nonoperative group versus $23 \%$ in the operative group with age $>70$ being a major risk factor. Our patient was elderly and presented with an unstable fracture of L1; therefore, surgery was performed. The use of the robotic-assisted device allowed us to perform only 6 fluoroscopic examinations (2 in the beginning of the operation for calibration, two for registration and two at the end of the operation in order to check the position of our fragments and hardware). A follow-up CT scan was not performed; thus, the position of the pedicular screws was assessed only by $\mathrm{X}$-ray.

The reasons for using the robot in this particular case included the clinical picture of instability (severe pain on every movement) as well as his underlying advanced age, AS and CLL. The patient had low hemoglobin (CLL) and would have been more susceptible to decompensation following a prolonged back surgery with significant blood loss due to his underlying hematologic condition as well as his older age and general condition. AS creates the expected difficulty in identifying the anatomy in the spine, further increasing the technical difficulty of the surgery and the likelihood of surgery-related complications. Utilizing the robot enables a relatively short operative period, with better precision and less complications, such as blood loss, infection and nonunion, which could be particularly significant in this specific patient.

We ultimately performed a shorter fusion than the standard long and rigid fixation that was originally planned for this patient. This is not the accepted approach to a fracture in a patient with AS. The decision was made based on the clinical impression during surgery of complete/rigid stability of all of the segments in the area following initial short segment stabilization. It was also made in the context of the high morbidity in this individual patient on one hand, and the low expected postoperative functional demand on the other. The patient healed, but this approach should not be established in any way as an appropriate treatment for fractures in patients with AS.
A disadvantage with the use of our system, which is CT-based, is that a preoperative scan is required. This is a disadvantage mostly in cases where a CT scan would not routinely be obtained prior to surgery, such as in scoliosis surgery. In our patient, a CT scan was necessary at any rate for the diagnosis and evaluation of the fracture in a spine with AS. Another disadvantage is the need for a manual registration, a process which is time consuming. This process has a learning curve and is therefore surgeon dependent. In this case, the total surgery time was 2 hours and we therefore feel that if there was added time due to the registration process, it may have been partially offset by the ease of screw placement.

There was a relatively limited amount of blood loss, and a short operative time. Postoperatively, there were no neurological deficits and no surgical complications. We believe that the use of a robotic assisted device is especially useful in cases with a high potential for complications.

\section{Conflict of Interest}

No potential conflict of interest relevant to this article was reported.

\section{References}

1. Bessant R, Keat A. How should clinicians manage osteoporosis in ankylosing spondylitis? J Rheumatol 2002;29:1511-9.

2. Caron T, Bransford R, Nguyen Q, Agel J, Chapman J, Bellabarba C. Spine fractures in patients with ankylosing spinal disorders. Spine (Phila Pa 1976) 2010;35:E458-64.

3. Kantelhardt SR, Martinez R, Baerwinkel S, Burger R, Giese A, Rohde V. Perioperative course and accuracy of screw positioning in conventional, open roboticguided and percutaneous robotic-guided, pedicle screw placement. Eur Spine J 2011;20:860-8.

4. Jo DJ, Kim SM, Kim KT, Seo EM. Surgical experience of neglected lower cervical spine fracture in patient with ankylosing spondylitis. J Korean Neurosurg Soc 2010;48:66-9. 